\title{
Uniform rotundity in every direction of Orlicz function spaces equipped with the $p$-Amemiya norm
}

\author{
Radosław Kaczmarek $^{1}$ (D)
}

Received: 5 June 2017 / Accepted: 13 March 2018 / Published online: 26 March 2018

(C) The Author(s) 2018

\begin{abstract}
Some conditions which guarantee that the Orlicz function spaces equipped with the p-Amemiya norm $(1<p<\infty)$ and generated by $\mathrm{N}$-functions are uniformly rotund in every direction are given. Obtained result broaden the knowledge about this notion in Orlicz function spaces with the $\mathrm{p}$-Amemiya norm $(1 \leq p \leq \infty)$.
\end{abstract}

Keywords Orlicz spaces · P-Amemiya norm · Uniform rotundity in every direction

Mathematics Subject Classification 46E30 - 46B20

\section{Introduction and preliminaries}

Let us denote by $\mathbb{N}, \mathbb{R}$ and $\mathbb{R}_{+}$the sets of natural, real and nonnegative real numbers, respectively. For a Banach space $X$, by $S(X)$ and $B(X)$ we will denote the unit sphere and the unit ball of $X$, respectively.

A Banach space $X=(X,\|\|$.$) is called uniformly rotund in every direction (see [16,42]$ ) if for each $\varepsilon>0$ and nonzero $z \in X$ there exists $\delta(z, \varepsilon)>0$ such that if $x$ and $y$ belong to $S(X),\|x-y\| \geq \varepsilon$ and $x-y=\alpha z$ for some $\alpha \in \mathbb{R}$, then $\left\|\frac{x+y}{2}\right\| \leq 1-\delta(z, \varepsilon)$. We will write then $X \in(U R E D)$ for short. It is known (see [16]) that the URED property is equivalent to the following one: For each nonzero $z$ in $X$ there is a positive number $\delta(z)$ such that if $x \in B(X)$ and $\|x+z\| \leq 1$, then $\left\|x+\frac{z}{2}\right\| \leq 1-\delta(z)$. Equivalently, one can say that $X \in(U R E D)$ if and only if $x_{n}, z \in X,\left\|x_{n}\right\| \rightarrow 1,\left\|x_{n}+z\right\| \rightarrow 1$ and $\left\|2 x_{n}+z\right\| \rightarrow 2$ as $n \rightarrow \infty$ imply $z=0$.

Another characterization of the uniform rotundity in every direction is also possible (see [16]), namely, $X \in(U R E D)$ if and only if the following condition holds: if there are sequences $\left(x_{n}\right)_{n=1}^{\infty}$ and $\left(y_{n}\right)_{n=1}^{\infty}$ in $X$ such that $\left\|x_{n}\right\| \leq 1$ and $\left\|y_{n}\right\| \leq 1$ for every $n \in \mathbb{N}$,

Radosław Kaczmarek

radekk@amu.edu.pl

1 Faculty of Mathematics and Computer Science, Adam Mickiewicz University in Poznań, Umultowska 87, 61-614 Poznan, Poland 
$x_{n}-y_{n} \rightarrow z$ and $\left\|x_{n}+y_{n}\right\| \rightarrow 2$ as $n \rightarrow \infty$, then $z=0$. Note also that in case of Köthe spaces, the following characterization of the URED property is known (see [32], Prop. 3.3): The Köthe space $E$ is uniformly rotund in every direction if and only if for any $\varepsilon \in(0,2]$ and $z \in E_{+} \backslash\{0\}\left(E_{+}\right.$is the positive cone of $\left.E\right)$ there exists $\delta(\varepsilon, z) \in(0,1)$ such that $\left\|\frac{x+y}{2}\right\|_{E} \leq 1-\delta(\varepsilon, z)$ for any $x, y \in B(E)$ with $x-y=\lambda z$ for some $\lambda>0$ and $\|x-y\|_{E} \geq \varepsilon$.

The notion of uniform rotundity in every direction was first used by Garkavi (see [1820]) to characterize normed linear spaces for which every bounded subset has at most one Chebyshev center. Next, Zizler, Day, James and Swaminathan continued investigation of this property $[16,46]$ and the revealed results and applications encouraged other mathematicians to consider this property in some particular Banach spaces such as Orlicz spaces, MusielakOrlicz spaces, Musielak-Orlicz spaces of Bochner type, Orlicz-Sobolev spaces or CalderónLozanovskiĭ spaces (see [2,4,5,8,27-29,32,41,43,44]).

A map $\Phi: \mathbb{R} \rightarrow[0, \infty]$ is said to be an Orlicz function if $\Phi(0)=0, \Phi$ is not identically equal to zero (i.e. $\lim _{u \rightarrow \infty} \Phi(u)=\infty$ ), $\Phi$ is even and convex on the interval $(-b(\Phi), b(\Phi)$ ) and $\Phi$ is left-continuous at $b(\Phi)$, i.e. $\lim _{u \rightarrow b(\Phi)^{-}} \Phi(u)=\Phi(b(\Phi))$. Let us notice that every Orlicz function $\Phi$ is continuous on the interval $(-b(\Phi), b(\Phi))$. Recall also that an Orlicz function $\Phi$ is called an $\mathrm{N}$-function if it vanishes only at 0 , takes only finite values and the following two conditions are satisfied: $\lim _{u \rightarrow 0} \frac{\Phi(u)}{u}=0$ and $\lim _{u \rightarrow \infty} \frac{\Phi(u)}{u}=\infty$.

Let us note that whenever some result will concern Orlicz spaces generated by $\mathrm{N}$-functions only, this fact will be announced in the assumptions.

For any Orlicz function $\Phi: \mathbb{R} \rightarrow[0, \infty]$ let us define

$$
a(\Phi):=\sup \{u \geq 0: \Phi(u)=0\}, b(\Phi):=\sup \{u>0: \Phi(u)<\infty\} .
$$

Notice that $a(\Phi)=0$ means that $\Phi$ vanishes only at zero while $b(\Phi)=\infty$ means that $\Phi$ takes only finite values.

An Orlicz function $\Phi: \mathbb{R} \rightarrow[0, \infty)$ is called convex if

$$
\Phi\left(\frac{u+v}{2}\right) \leq \frac{\Phi(u)+\Phi(v)}{2}
$$

for all $u, v \in \mathbb{R}$. If the inequality in (1.1) is sharp for all $u \neq v$, then $\Phi$ is called strictly convex (on $\mathbb{R}$ ). An Orlicz function $\Phi: \mathbb{R} \rightarrow[0, \infty)$ is said to be uniformly convex at infinity (see also [1,34]) if for each $a \in(0,1)$ there exists $\delta_{a} \in(0,1)$ such that

$$
\Phi\left(\frac{u+a u}{2}\right) \leq \frac{1}{2}\left(1-\delta_{a}\right)(\Phi(u)+\Phi(a u))
$$

for all $u \geq u_{0}$, where $u_{0}>0$. If condition (1.2) holds with $u_{0}=0$, we say that $\Phi$ is uniformly convex on $\mathbb{R}_{+}$(so on the whole $\mathbb{R}$ by the fact that $\Phi$ is even).

For any Orlicz function $\Phi$, we define its complementary (in the sense of Young) function $\Psi$ by the formula

$$
\Psi(v)=\sup _{u \geq 0}\{u|v|-\Phi(u)\}
$$

We say that an Orlicz function $\Phi$ satisfies condition $\Delta_{2}\left(\mathbb{R}_{+}\right)$[resp. $\left.\Delta_{2}(\infty)\right]$ if there exists $K>0$ such that for all $u \geq 0$ [resp. if there exist $K>0$ and $u_{0}>0$ with $\Phi\left(u_{0}\right)<\infty$ such that for any $\left.u \geq u_{0}\right]$ inequality $\Phi(2 u) \leq K \Phi(u)$ holds. In this case, we will write $\Phi \in \Delta_{2}\left(\mathbb{R}_{+}\right)\left[\right.$resp. $\left.\Phi \in \Delta_{2}(\infty)\right]$.

Throughout the paper we will assume that $(\Omega, \Sigma, \mu)$ is a measure space with a $\sigma$-finite non-atomic and complete measure $\mu$ and $L^{0}(\mu)$ is the space of all $\mu$-equivalence classes of 
real and $\Sigma$-measurable functions defined on $\Omega$. Let us note that Theorem 4 will be formulated for the Orlicz spaces equipped with the p-Amemiya norm, $(1 \leq p<\infty)$, built over a finite non-atomic measure space. We shall say that an Orlicz function $\Phi$ satisfies the $\Delta_{2}(\mu)$ condition if $\Phi \in \Delta_{2}(\infty)$ when the measure space is non-atomic finite and $\Phi \in \Delta_{2}\left(\mathbb{R}_{+}\right)$ when the measure space is non-atomic infinite. Let us define the characteristic function $\chi_{A}$ of a subset $A$ from $\Omega$ as

$$
\chi_{A}= \begin{cases}1, & \text { for } t \in A, \\ 0, & \text { otherwise. }\end{cases}
$$

For a given Orlicz function $\Phi$ we define on $L^{0}(\mu)$ a convex semimodular (see $[3,30,34$ $36,39])$ by

$$
I_{\Phi}(x)=\int_{\Omega} \Phi(x(t)) d \mu .
$$

The Orlicz space $L^{\Phi}$ generated by an Orlicz function $\Phi$ is a linear space of measurable functions defined by the formula (see [38]):

$$
L^{\Phi}=\left\{x \in L^{0}(\mu): I_{\Phi}(\lambda x)<\infty \text { for some } \lambda>0\right\} .
$$

The Orlicz space is usually equipped with the Luxemburg norm (see [34])

$$
\|x\|_{\Phi}=\inf \left\{\lambda>0: I_{\Phi}\left(\frac{x}{\lambda}\right) \leq 1\right\}
$$

or with the equivalent one (see $[37,38]$ )

$$
\|x\|_{o}=\sup \left\{\int_{\Omega}|x(t) y(t)| d \mu: y \in L^{\Psi}, I_{\Psi}(y) \leq 1\right\},
$$

which is called the Orlicz norm, where $\Psi$ is the complementary function to $\Phi$.

For any $1 \leq p \leq \infty$ and $u \geq 0$ let us define

$$
s_{p}(u)= \begin{cases}\left(1+u^{p}\right)^{\frac{1}{p}} & \text { for } 1 \leq p<\infty \\ \max \{1, u\} & \text { for } p=\infty\end{cases}
$$

In order to simplify notations, define $s_{\Phi, p}(x)=s_{p} \circ I_{\Phi}(x)$ for all $1 \leq p \leq \infty$ and all $x \in L^{0}(\mu)$. Note that the functions $s_{p}$ and $s_{\Phi, p}$ are convex. Moreover, the function $s_{p}$ is increasing on $\mathbb{R}_{+}$for $1 \leq p<\infty$, but the function $s_{\infty}$ is increasing on the interval $[1, \infty)$ only.

Definition 1 Let $p \in[1, \infty]$. By the p-Amemiya norm of a function $x \in L^{0}$ we mean the number defined by the formula (see $[11,25]$ )

$$
\|x\|_{\Phi, p}=\inf _{k>0} \frac{1}{k} s_{\Phi, p}(k x) .
$$

The Orlicz space equipped with the p-Amemiya norm $\left(L^{\Phi},\|\cdot\|_{\Phi, p}\right)$ will be denoted by $L^{\Phi, p}$. It is known (see [11]) that the p-Amemiya norm $\|x\|_{\Phi, p}(1 \leq p \leq \infty)$ is equivalent to the Luxemburg norm $\|x\|_{\Phi}$, namely, $\|x\|_{\Phi} \leq\|x\|_{\Phi, p} \leq 2^{\frac{1}{p}}\|x\|_{\Phi}$ for any $x \in L^{\Phi, p}$. Recall also here the earlier result of Hudzik and Maligranda from [25], which states that the 1-Amemiya norm is equal to the Orlicz norm in general, i.e. when $\Phi$ is an arbitrary Orlicz function.

Recall that the notion of the p-Amemiya norm $(1 \leq p \leq \infty)$ was introduced by Reisner in 1988 in [40], where the Author defined these norms for the Calderón-Lozanovskii spaces. 
Next, in 2000, Hudzik and Maligranda [25] suggested investigating a family of p-Amemiya norms $(1 \leq p \leq \infty)$ in Orlicz spaces. After several years, the first paper constituting some basic and crutial results allowing further research and containing the complete characterization of rotundity and extreme points in Orlicz spaces equipped with the p-Amemiya norms, $1 \leq p \leq \infty$, was written by Cui et al. (see [11]). Since that time, an intensive development of research connected with Orlicz and Musielak-Orlicz spaces equipped with the p-Amemiya norm has taken place, many important results broaden the knowledge about the geometry of these spaces were obtained (see [6,7,9-15,17,23,24,26,33]) and some open questions were put (see [45]).

Up to the end of this section, let $p \in[1, \infty]$. Denoting by $p_{+}$the right-side derivative of the function $\Phi$ on $[0, b(\Phi))$ and putting $p_{+}(b(\Phi))=\lim _{u \rightarrow b(\Phi)^{-}} p_{+}(u)$, let us define the function $\alpha_{p}: L^{\Phi, p} \rightarrow[-1, \infty]$ by

$$
\alpha_{p}(x)= \begin{cases}I_{\Phi}^{p-1}(x) I_{\Psi}\left(p_{+}(|x|)\right)-1, & \text { if } 1 \leq p<\infty \\ -1, & \text { if } p=\infty \wedge I_{\Phi}(x) \leq 1, \\ I_{\Psi}\left(p_{+}(|x|)\right), & \text { if } p=\infty \wedge I_{\Phi}(x)>1,\end{cases}
$$

and the functions $k_{p}^{*}: L^{\Phi, p} \rightarrow[0, \infty), k_{p}^{* *}: L^{\Phi, p} \rightarrow(0, \infty]$ by

$$
\begin{aligned}
& k_{p}^{*}(x)=\inf \left\{k \geq 0: \alpha_{p}(k x) \geq 0\right\} \quad(\text { with } \inf \emptyset=\infty), \\
& k_{p}^{* *}(x)=\sup \left\{k \geq 0: \alpha_{p}(k x) \leq 0\right\} .
\end{aligned}
$$

It is obvious that $k_{p}^{*}(x) \leq k_{p}^{* *}(x)$ for every $1 \leq p \leq \infty$ and $x \in L^{\Phi, p}$.

Denote by $K_{p}(x)$ the set of all $k \in(0, \infty)$ which are between $k_{p}^{*}(x)$ and $k_{p}^{* *}(x)$, i.e., $K_{p}(x)=\left\{0<k<\infty: k_{p}^{*}(x) \leq k \leq k_{p}^{* *}(x)\right\}$. Note that $K_{p}(x)=\varnothing$ if and only if $k_{p}^{*}(x)=k_{p}^{* *}(x)=\infty$. Moreover, the p-Amemiya norm $\|x\|_{\Phi, p}, x \neq 0$, is attained at every point $k \in\left[k_{p}^{*}(x), k_{p}^{* *}(x)\right)$ provided $k_{p}^{*}(x)<\infty$ and at every point $k \in\left[k_{p}^{*}(x), k_{p}^{* *}(x)\right]$ provided $k_{p}^{* *}(x)<\infty$ (see [11]). Recall also that an Orlicz function $\Phi$ is said to be $k_{p}^{*}$-finite (respectively $k_{p}^{* *}$-finite) provided $k_{p}^{*}(x)<\infty$ (resp. $k_{p}^{* *}(x)<\infty$ ) for every $x \in L^{\Phi, p}$. An Orlicz function $\Phi$ is said to be $k_{p}$-unique, if $0<k_{p}^{*}(x)=k_{p}^{* *}(x)<\infty$ for all $x \in L^{\Phi, p} \backslash\{0\}$. Evidently, $\Phi$ is $k_{p}$-unique if and only if card $K_{p}(x)=1$ for every $x \in L^{\Phi, p} \backslash\{0\}$.

\section{Auxiliary results}

Let us first note that although the main theorem is formulated for the Orlicz spaces generated by $\mathrm{N}$-functions only, we will present some auxiliary results in they original and often wider form, i.e. formulated for Orlicz spaces generated by an arbitrary Orlicz function.

Lemma 1 (See [3], Proposition 1.4) Let $\Phi$ be a strictly convex $N$-function. Then:

(1) $\Phi$ is uniformly convex on any bounded interval.

(2) For any $K>0, \varepsilon>0$ and $[a, b] \subset(0,1)$, there exists $\delta>0$ such that

$$
\Phi(\alpha u+(1-\alpha) v) \leq(1-\delta)[\alpha \Phi(u)+(1-\alpha) \Phi(v))]
$$

holds for all $\alpha \in[a, b]$ and $u, v \in \mathbb{R}$ satisfying $|u|,|v| \leq K$ and $|u-v| \geq \varepsilon$.

Theorem 1 (Ergoroff's theorem, see [22]) If $f_{n}$ and $f$ are measurable and almost everywhere finite in $\Omega, \mu(\Omega)<\infty$ and $f_{n}(t) \rightarrow f(t)$ a.e. in $\Omega$, then for every number $\varepsilon>0$ there exists a set $A \in \Sigma$ such that $\mu(A)<\varepsilon$ and $f_{n}(t) \rightarrow f(t)$ uniformly in $\Omega \backslash A$. 
Theorem 2 (See [11], Theorem 6.2) The Orlicz space $L^{\Phi, p}=\left(L^{\Phi},\|\cdot\|_{\Phi, p}\right)$ is rotund if and only if

1. $\Phi$ is $k_{p}$-unique and

2. $\Phi$ is strictly convex on $(-b(\Phi), b(\Phi))$ and

3. (a) $1 \leq p<\infty$ or

(b) $p=\infty$ and $\Phi \in \Delta_{2}(\mu)$.

Corollary 1 (See [11], Corollary 4.7) Every Orlicz function $\Phi$ that is strictly convex on $(-b(\Phi), b(\Phi))$ is $k_{p}$-unique for all $1<p \leq \infty$. Moreover, if $b(\Phi)=\infty$ and $\Phi$ does not have an asymptote at $\infty$, then $\Phi$ is $k_{p}$-unique for all $1 \leq p \leq \infty$.

Lemma 2 (See [15], Lemma 2.7) Let $1 \leq p \leq \infty$. Then $I_{\Phi}(x) \leq\|x\|_{\Phi, p}$ for all $x \in L^{\Phi, p}$ with $\|x\|_{\Phi, p} \leq 1$.

Lemma 3 (See [15], Lemma 4.2) Let $1 \leq p \leq \infty$ and let $\Phi$ be an Orlicz function. In the case $p=1$ assume, additionally, that $\Phi$ is $k_{1}^{*}$-finite. If the sequence $\left(\left\|x_{n}\right\|_{\Phi, p}\right)_{n=1}^{\infty}$ is bounded, $k_{n} \in K_{p}\left(x_{n}\right)$ and $k_{n} \rightarrow \infty$, then $x_{n} \stackrel{\mu}{\rightarrow} 0$ on $\Omega$.

Lemma 4 (See [44], Lemma 6 or [3], Lemma 2.35) Let $\Phi$ be an $N$-function. Then, for a given $\sigma \in\left(0, \frac{1}{2}\right), \varepsilon>0$ and $\gamma>0$, there exists $\delta>0$ such that for any $u>0$ and any $\lambda \in\left[\sigma, \frac{1}{2}\right]$ satisfying

$$
\lambda \Phi((1+\varepsilon) u)+(1-\lambda) \Phi(u) \leq(1+\delta)[\Phi(\lambda(1+\varepsilon) u+(1-\lambda) u)],
$$

there exists $\tau \in[(1+\lambda \varepsilon) u,(1+\varepsilon) u]$ such that

$$
p_{+}(\tau) \leq(1+\gamma) p_{+}\left(\frac{1+\sigma \varepsilon}{1+2 \sigma \varepsilon} \tau\right) .
$$

Recall that in 1984 Kamińska (see [27]) gave the characterization of the uniform rotundity in every direction for Musielak-Orlicz spaces endowed with the Luxemburg norm over a nonatomic measure space, which was next generalized to the Musielak-Orlicz spaces of Bochner type (see [28]). The theorem presented below can be easily deduced from the result contained in [27]. Let us also note that another proof of the characterization of the URED property for Orlicz spaces equipped with the Luxemburg norm was given by Hudzik in [8].

Theorem 3 (See [27] or [31], Chapter 12) Let $(\Omega, \Sigma, \mu)$ be a non-atomic complete and $\sigma$-finite measure space and $\Phi: \mathbb{R} \rightarrow \mathbb{R}_{+}$be an even, continuous, convex and vanishing only at zero function. Then the Orlicz space $\left(L^{\Phi},\|\cdot\|_{\Phi}\right)$ equipped with the Luxemburg norm is uniformly rotund in every direction if and only if $\Phi$ is strictly convex and $\Phi$ satisfies the $\Delta_{2}(\mu)$-condition.

\section{Results}

Denote $\ell_{2}^{p}=\left(\mathbb{R}^{2},\|\cdot\|_{p}\right)$, where the norm $\|\cdot\|_{p}$ is defined as $\|x\|_{p}=\left(\left|x_{1}\right|^{p}+\left|x_{2}\right|^{p}\right)^{1 / p}$ for any $x=\left(x_{1}, x_{2}\right) \in \mathbb{R}^{2}$ and any $p \in[1, \infty)$, and $\|x\|_{\infty}=\max \left\{\left|x_{1}\right|,\left|x_{2}\right|\right\}$, whenever $p=\infty$. Then, the p-Amemiya norm $(1 \leq p<\infty)$ in the Orlicz space $L^{\Phi, p}$ can be expressed by the help of the norm $\|\cdot\|_{p}$ in the following way:

$$
\|x\|_{\Phi, p}=\inf _{k>0}\left\|\left(1, I_{\Phi}(k x)\right)\right\|_{p} .
$$


For $u \in\left(L^{\Phi},\|\cdot\|_{\Phi, p}\right)$ and $v \in\left(L^{\Psi},\|\cdot\|_{\Psi, q}\right)$, where $p, q \in[1, \infty]$ are such that $\frac{1}{p}+\frac{1}{q}=1$, let $\langle v, u\rangle:=\int_{\Omega} u(t) v(t) d t$.

Let us start from presenting some lemmas. The first one is formulated for the Orlicz spaces generated by $N$-functions and endowed with the p-Amemiya norm but only for $p \in(1, \infty)$ (for the case $p=1$, we refer to [44] or [3]).

Lemma 5 Let $\Phi$ be an $N$-function, $\Psi$ be its complementary in the sense of Young function and let $p \in(1, \infty)$ and $q \in(1, \infty)$ be such numbers that $\frac{1}{p}+\frac{1}{q}=1$. If $x_{n}, y_{n} \in$ $B\left(L^{\Phi, p}\right)$ and $v_{n} \in B\left(L^{\Psi, q}\right)$ satisfy $\left\langle v_{n}, x_{n}+y_{n}\right\rangle \rightarrow 2$ as $n \rightarrow \infty$, then for any $\Omega_{n} \in \Sigma$, $k_{n} \in K_{p}\left(x_{n}\right)$ and $h_{n} \in K_{p}\left(y_{n}\right)$, we have that $\lim _{n \rightarrow \infty} \int_{\Omega}\left[k_{n} x_{n}(t)-h_{n} y_{n}(t)\right] v_{n}(t) d t=$ $\lim _{n \rightarrow \infty}\left[\left\|k_{n} x_{n}\right\|_{\Phi, p}-\left\|h_{n} y_{n}\right\|_{\Phi, p}\right]=\lim _{n \rightarrow \infty}\left[\left(1+I_{\Phi}^{p}\left(k_{n} x_{n}\right)\right)^{\frac{1}{p}}-\left(1+I_{\Phi}^{p}\left(h_{n} y_{n}\right)\right)^{\frac{1}{p}}\right]$ and

$$
\lim _{n \rightarrow \infty} \int_{\Omega_{n}}\left[k_{n} x_{n}(t)-h_{n} y_{n}(t)\right] v_{n}(t) d t \leq \lim _{n \rightarrow \infty}\left|I_{\Phi}\left(k_{n} x_{n} \chi_{\Omega_{n}}\right)-I_{\Phi}\left(h_{n} y_{n} \chi_{\Omega_{n}}\right)\right|
$$

hold provided that the limits exist and $\left\{\max \left\{k_{n}, h_{n}\right\}\right\}_{n}$ is bounded.

Proof Let $p \in(1, \infty)$. By the assumption, we get that $\left\langle v_{n}, x_{n}\right\rangle \rightarrow 1$ and $\left\langle v_{n}, y_{n}\right\rangle \rightarrow 1$ as $n \rightarrow \infty$, so by the Hölder inequality

$$
\begin{aligned}
1 & \leftarrow\left\langle v_{n}, x_{n}\right\rangle=\int_{\Omega} v_{n}(t) x_{n}(t) d t \leq\left\|v_{n}\right\|_{\Psi, q} \cdot\left\|x_{n}\right\|_{\Phi, p} \leq\left\|x_{n}\right\|_{\Phi, p} \\
& =\frac{1}{k_{n}}\left(1+I_{\Phi}^{p}\left(k_{n} x_{n}\right)\right)^{\frac{1}{p}} \leq 1,
\end{aligned}
$$

whence

$$
\left(1+I_{\Phi}^{p}\left(k_{n} x_{n}\right)\right)^{\frac{1}{p}}-\int_{\Omega} k_{n} x_{n}(t) v_{n}(t) d t \rightarrow 0
$$

as $n \rightarrow \infty$. In a similar way we can prove that

$$
\left(1+I_{\Phi}^{p}\left(h_{n} y_{n}\right)\right)^{\frac{1}{p}}-\int_{\Omega} h_{n} y_{n}(t) v_{n}(t) d t \rightarrow 0
$$

as $n \rightarrow \infty$. Formulas (3.2) and (3.3) yield

$$
\lim _{n \rightarrow \infty} \int_{\Omega}\left[k_{n} x_{n}(t)-h_{n} y_{n}(t)\right] v_{n}(t) d t=\lim _{n \rightarrow \infty}\left[\left(1+I_{\Phi}^{p}\left(k_{n} x_{n}\right)\right)^{\frac{1}{p}}-\left(1+I_{\Phi}^{p}\left(h_{n} y_{n}\right)\right)^{\frac{1}{p}}\right],
$$

whence

$$
\begin{aligned}
\lim _{n \rightarrow \infty} \int_{\Omega}\left[k_{n} x_{n}(t)-h_{n} y_{n}(t)\right] v_{n}(t) d t & =\lim _{n \rightarrow \infty}\left[\left\|k_{n} x_{n}\right\|_{\Phi, p}-\left\|h_{n} y_{n}\right\|_{\Phi, p}\right] \\
& \leq \lim _{n \rightarrow \infty}\left|\left\|\left(1, I_{\Phi}\left(k_{n} x_{n}\right)\right)\right\|_{p}-\left\|\left(1, I_{\Phi}\left(h_{n} y_{n}\right)\right)\right\|_{p}\right| \\
& \leq \lim _{n \rightarrow \infty}\left\|\left(0, I_{\Phi}\left(k_{n} x_{n}\right)-I_{\Phi}\left(h_{n} y_{n}\right)\right)\right\|_{p} \\
& =\lim _{n \rightarrow \infty}\left|I_{\Phi}\left(k_{n} x_{n}\right)-I_{\Phi}\left(h_{n} y_{n}\right)\right|,
\end{aligned}
$$


so for any $\Omega_{n} \in \Sigma$, we obtain

$$
\lim _{n \rightarrow \infty} \int_{\Omega_{n}}\left[k_{n} x_{n}(t)-h_{n} y_{n}(t)\right] v_{n}(t) d t \leq \lim _{n \rightarrow \infty}\left|I_{\Phi}\left(k_{n} x_{n} \chi_{\Omega_{n}}\right)-I_{\Phi}\left(h_{n} y_{n} \chi_{\Omega_{n}}\right)\right|
$$

and the proof is finished.

Let us now present the more general result rather then this presented in [44], Lemma 4 or in [3], Lemma 2.26.

Lemma 6 Let $\Phi$ be a strictly convex $N$-function, let $p \in[1, \infty), x_{n}, y_{n} \in B\left(L^{\Phi, p}\right), \| x_{n}+$ $y_{n} \|_{\Phi, p} \rightarrow 2$ as $n \rightarrow \infty, k_{n} \in K_{p}\left(x_{n}\right)$ and $h_{n} \in K_{p}\left(y_{n}\right)$. Then $b=\sup \max \left\{k_{n}, h_{n}\right\}<\infty$ implies $k_{n} x_{n}-h_{n} y_{n} \rightarrow 0$ in measure.

Proof Recall first that an Orlicz function $\Phi$ which vanishes only at zero and takes only finite values has the following property:

$$
\Phi(|u|-|v|) \leq|\Phi(2 u)-\Phi(2 v)| \text { for all } u, v \in \mathbb{R} .
$$

Notice that for any $p \in[1, \infty)$, the function $f(u):=s_{p}(|u|)-1, u \in \mathbb{R}$, is an Orlicz function, so by property (3.4) applied to the $f$, i.e. $f\left(\frac{1}{2}|u|-\frac{1}{2}|v|\right) \leq|f(u)-f(v)|$ for all $u, v \in \mathbb{R}$, as well as by the convexity of the function $s_{p}($.), we get

$$
\begin{aligned}
& 0 \leftarrow 2-\left\|x_{n}+y_{n}\right\|_{\Phi, p} \geq\left\|x_{n}\right\|_{\Phi, p}+\left\|y_{n}\right\|_{\Phi, p}-\left\|x_{n}+y_{n}\right\|_{\Phi, p} \\
& \geq \frac{1}{k_{n}} s_{\Phi, p}\left(k_{n} x_{n}\right)+\frac{1}{h_{n}} s_{\Phi, p}\left(h_{n} y_{n}\right)-\frac{1}{\frac{k_{n} h_{n}}{k_{n}+h_{n}}} s_{\Phi, p}\left(\frac{k_{n} h_{n}}{k_{n}+h_{n}}\left(x_{n}+y_{n}\right)\right) \\
& =\frac{k_{n}+h_{n}}{k_{n} h_{n}}\left\{\frac{h_{n} s_{p}\left(I_{\Phi}\left(k_{n} x_{n}\right)\right)}{k_{n}+h_{n}}+\frac{k_{n} s_{p}\left(I_{\Phi}\left(h_{n} y_{n}\right)\right)}{k_{n}+h_{n}}-s_{p}\left(I_{\Phi}\left(\frac{k_{n} h_{n}}{k_{n}+h_{n}}\left(x_{n}+y_{n}\right)\right)\right)\right\} \\
& \geq \frac{k_{n}+h_{n}}{k_{n} h_{n}}\left\{s_{p}\left(\frac{h_{n} I_{\Phi}\left(k_{n} x_{n}\right)}{k_{n}+h_{n}}+\frac{k_{n} I_{\Phi}\left(h_{n} y_{n}\right)}{k_{n}+h_{n}}\right)-s_{p}\left(I_{\Phi}\left(\frac{k_{n} h_{n}}{k_{n}+h_{n}}\left(x_{n}+y_{n}\right)\right)\right)\right\} \\
& =\frac{k_{n}+h_{n}}{k_{n} h_{n}}\left\{s_{p}\left(\frac{h_{n} I_{\Phi}\left(k_{n} x_{n}\right)}{k_{n}+h_{n}}+\frac{k_{n} I_{\Phi}\left(h_{n} y_{n}\right)}{k_{n}+h_{n}}\right)-1-\left[s_{p}\left(I_{\Phi}\left(\frac{k_{n} h_{n}}{k_{n}+h_{n}}\left(x_{n}+y_{n}\right)\right)\right)-1\right]\right\} \\
& \geq \frac{k_{n}+h_{n}}{k_{n} h_{n}}\left\{s_{p}\left[\frac{1}{2}\left(\frac{h_{n} I_{\Phi}\left(k_{n} x_{n}\right)}{k_{n}+h_{n}}+\frac{k_{n} I_{\Phi}\left(h_{n} y_{n}\right)}{k_{n}+h_{n}}-I_{\Phi}\left(\frac{k_{n} h_{n}}{k_{n}+h_{n}}\left(x_{n}+y_{n}\right)\right)\right)\right]-1\right\} \geq 0,
\end{aligned}
$$

so

$$
s_{p}\left[\frac{1}{2}\left(\frac{h_{n} I_{\Phi}\left(k_{n} x_{n}\right)}{k_{n}+h_{n}}+\frac{k_{n} I_{\Phi}\left(h_{n} y_{n}\right)}{k_{n}+h_{n}}-I_{\Phi}\left(\frac{k_{n} h_{n}}{k_{n}+h_{n}}\left(x_{n}+y_{n}\right)\right)\right)\right] \rightarrow 1
$$

as $n \rightarrow \infty$. Since $s_{p}\left(u_{n}\right) \rightarrow 1$ if and only if $u_{n} \rightarrow 0$ as $n \rightarrow \infty$, we get that

$$
\frac{h_{n} I_{\Phi}\left(k_{n} x_{n}\right)}{k_{n}+h_{n}}+\frac{k_{n} I_{\Phi}\left(h_{n} y_{n}\right)}{k_{n}+h_{n}}-I_{\Phi}\left(\frac{k_{n} h_{n}}{k_{n}+h_{n}}\left(x_{n}+y_{n}\right)\right) \rightarrow 0
$$

as $n \rightarrow \infty$.

If the sequence $\left(k_{n} x_{n}-h_{n} y_{n}\right)_{n=1}^{\infty}$ does not converge to zero in measure, then without loss of generality, we may assume that $\mu\left(E_{n}\right)>\varepsilon$ for any $n \in \mathbb{N}$, where $E_{n}=\{t \in \Omega$ : $\left.\left|k_{n} x_{n}(t)-h_{n} y_{n}(t)\right| \geq \sigma\right\}$ and $\sigma, \varepsilon$ are fixed and positive numbers.

Since the Orlicz space $L^{\Phi, p}$ is a symmetric space, the norm $\left\|\chi_{F}\right\|_{\Phi, p}$ does not depend on the set $F$ but only on its measure, so let us denote $k=\frac{1}{\left\|\chi_{F}\right\|_{\Phi, p}}$, where $F \in \Sigma$ and $\mu(F)=\frac{\varepsilon}{4}$. Define the sets

$$
A_{n}=\left\{t \in \Omega:\left|x_{n}(t)\right|>k\right\}, B_{n}=\left\{t \in \Omega:\left|y_{n}(t)\right|>k\right\} .
$$


Then $1 \geq\left\|x_{n}\right\|_{\Phi, p} \geq\left\|x_{n} \chi_{A_{n}}\right\|_{\Phi, p}>k\left\|\chi_{A_{n}}\right\|_{\Phi, p}$, whence $\left\|\chi_{A_{n}}\right\|_{\Phi, p}<\frac{1}{k}$, which implies $\mu\left(A_{n}\right)<\frac{\varepsilon}{4}$. In a similar way we get that $\mu\left(B_{n}\right)<\frac{\varepsilon}{4}$. By Lemma 1 , there exists $\delta>0$ such that

$$
\Phi(\alpha u+(1-\alpha) v) \leq(1-\delta)[\alpha \Phi(u)+(1-\alpha) \Phi(v)]
$$

holds for all $\alpha \in\left[\frac{1}{1+b}, \frac{b}{1+b}\right] \in(0,1)$ and any $u, v \in \mathbb{R}$ with $|u| \leq b k,|v| \leq b k$ and $|u-v| \geq \sigma$. Since $\frac{k_{n}}{k_{n}+h_{n}}, \frac{h_{n}}{k_{n}+h_{n}} \in\left[\frac{1}{1+b}, \frac{b}{1+b}\right]$ for all $t \in F_{n}:=E_{n} \backslash\left\{A_{n} \cup B_{n}\right\}$, we obtain that

$$
\Phi\left(\frac{k_{n} h_{n}\left(x_{n}(t)+y_{n}(t)\right)}{k_{n}+h_{n}}\right) \leq(1-\delta)\left[\frac{h_{n} \Phi\left(k_{n} x_{n}(t)\right)}{k_{n}+h_{n}}+\frac{k_{n} \Phi\left(h_{n} y_{n}(t)\right)}{k_{n}+h_{n}}\right] .
$$

By the convexity of $\Phi$ and condition (3.5), we get that

$$
\begin{aligned}
& 0 \leftarrow \frac{h_{n} I_{\Phi}\left(k_{n} x_{n}\right)}{k_{n}+h_{n}}+\frac{k_{n} I_{\Phi}\left(h_{n} y_{n}\right)}{k_{n}+h_{n}}-I_{\Phi}\left(\frac{k_{n} h_{n}}{k_{n}+h_{n}}\left(x_{n}+y_{n}\right)\right) \\
& \geq \frac{h_{n} I_{\Phi}\left(k_{n} x_{n} \chi_{F_{n}}\right)}{k_{n}+h_{n}}+\frac{k_{n} I_{\Phi}\left(h_{n} y_{n} \chi_{F_{n}}\right)}{k_{n}+h_{n}}-I_{\Phi}\left(\frac{k_{n} h_{n}}{k_{n}+h_{n}}\left(x_{n}+y_{n}\right) \chi_{F_{n}}\right) \\
& \geq \frac{h_{n}}{k_{n}+h_{n}} I_{\Phi}\left(k_{n} x_{n} \chi_{F_{n}}\right)+\frac{k_{n}}{k_{n}+h_{n}} I_{\Phi}\left(h_{n} y_{n} \chi_{F_{n}}\right) \\
&\left.\left.-(1-\delta)\left[\frac{h_{n}}{k_{n}+h_{n}} I_{\Phi}\left(k_{n} x_{n} \chi_{F_{n}}\right)+\frac{k_{n}}{k_{n}+h_{n}} I_{\Phi}\left(h_{n} y_{n} \chi_{F_{n}}\right)\right] \text { by (3. }\right)\right] \\
&= \delta\left[\frac{h_{n}}{k_{n}+h_{n}} I_{\Phi}\left(k_{n} x_{n} \chi_{F_{n}}\right)+\frac{k_{n}}{k_{n}+h_{n}} I_{\Phi}\left(h_{n} y_{n} \chi_{F_{n}}\right)\right] \\
& \geq \delta\left[\frac{1}{2 b} I_{\Phi}\left(k_{n} x_{n} \chi_{F_{n}}\right)+\frac{1}{2 b} I_{\Phi}\left(h_{n} y_{n} \chi_{F_{n}}\right)\right] \\
&= \delta\left(\frac{1}{2 b} I_{\Phi}\left(k_{n} x_{n} \chi_{F_{n}}\right)+\frac{1}{2 b} I_{\Phi}\left(-h_{n} y_{n} \chi_{F_{n}}\right)\right) \\
&= \frac{\delta}{b}\left(\frac{I_{\Phi}\left(k_{n} x_{n} \chi_{F_{n}}\right)+I_{\Phi}\left(-h_{n} y_{n} \chi_{F_{n}}\right)}{2}\right) \\
& \geq \frac{\delta}{b} I_{\Phi}\left(\frac{\left(k_{n} x_{n}-h_{n} y_{n}\right) \chi_{F_{n}}}{2}\right) \geq \frac{\delta}{b} \int\left(\frac{\sigma}{2}\right) d t \\
& \geq \frac{\delta}{b} \Phi\left(\frac{\sigma}{2}\right) \frac{\varepsilon}{2}, \\
&\left.E_{n} \cup B_{n}\right\}
\end{aligned}
$$

where $\sigma, \varepsilon>0$ were fixed, a contradiction.

Lemma 7 Let $(X,\|\|$.$) be a Banach space. If x_{n}, y_{n} \in B(X)$ for any $n \in \mathbb{N}$ and $\left\|\frac{x_{n}+y_{n}}{2}\right\| \rightarrow$ 1 , then $\left\|\alpha x_{n}+(1-\alpha) y_{n}\right\| \rightarrow 1$ for any $\alpha \in(0,1)$ as $n \rightarrow \infty$.

Proof Assume that $x_{n}, y_{n} \in B(X)$ for any $n \in \mathbb{N}$ and $\left\|\frac{x_{n}+y_{n}}{2}\right\| \rightarrow 1$, but there exists $\alpha \in(0,1)$ such that $\left\|\alpha x_{n}+(1-\alpha) y_{n}\right\| \nrightarrow 1$ as $n \rightarrow \infty$. Then, we can assume that there exists $\varepsilon \in(0,1)$ such that $\left\|\alpha x_{n}+(1-\alpha) y_{n}\right\| \leq 1-\varepsilon$. Denote $z_{n, \alpha}=\alpha x_{n}+(1-\alpha) y_{n}$. Then, we can find:

case a) either $\beta \in(0,1)$ such that $\frac{x_{n}+y_{n}}{2}=\beta z_{n, \alpha}+(1-\beta) y_{n}$ or case b) $\widetilde{\beta} \in(0,1)$ such that $\frac{x_{n}+y_{n}}{2}=\widetilde{\beta} x_{n}+(1-\widetilde{\beta}) z_{n, \alpha}$, where $z_{n, \alpha}$ is generated either by some $\alpha \in\left(\frac{1}{2}, 1\right)$ or some $\alpha \in\left(0, \frac{1}{2}\right)$, respectively. Since the proof for case b) is similar, it is omitted. Consequently 


$$
1 \leftarrow\left\|\frac{x_{n}+y_{n}}{2}\right\| \leq \beta\left\|z_{n, \alpha}\right\|+(1-\beta)\left\|y_{n}\right\| \leq \beta(1-\varepsilon)+1-\beta=1-\beta \varepsilon,
$$

a contradiction.

Let $\Phi$ be an N-function, $\Psi$ be its complementary (in the sense of Young) function, let $p_{+}$ be the right derivative of $\Phi$ and $q_{+}$be the right-inverse function of $p_{+}$, that is,

$$
q_{+}(s)=\sup \left\{t: p_{+}(t) \leq s\right\}=\inf \left\{t: p_{+}(t)>s\right\} .
$$

Now, we will present the sufficient conditions for the uniform rotundity in every direction in Orlicz spaces equipped with the $\mathrm{p}$-Amemiya norm, where $p \in[1, \infty)$. Although Theorem 4 is formulated for Orlicz spaces endowed with the $\mathrm{p}$-Amemiya norm, $p \in[1, \infty)$, generated by $\mathrm{N}$-functions and built over a non-atomic finite measure space, we will present its proof only in case of the Orlicz spaces equipped with the $\mathrm{p}$-Amemiya norm, $p \in(1, \infty)$, because in case of the Orlicz spaces endowed with the Orlicz norm, the proof can be found in [44] or [3].

Theorem 4 Let $(\Omega, \Sigma, \mu)$ be a non-atomic finite and complete measure space and let $p \in$ $[1, \infty)$. If

(a) $\Phi$ is a strictly convex $N$-function,

(b) for any $u_{0}>0, \varepsilon>0$ and $\widetilde{\varepsilon}>0$ there exist $\gamma>0$ and $A>0$ such that for any $u \geq u_{0}$, if $p_{+}((1+\varepsilon) u) \leq(1+\gamma) p_{+}(u)$, then $p_{+}(u) \leq A p_{+}(\widetilde{\varepsilon} u)$,

then the Orlicz space $\left(L^{\Phi},\|\cdot\|_{\Phi, p}\right)$ equipped with the p-Amemiya norm and generated by an $N$-function $\Phi$ is uniformly rotund in every direction.

Proof In the whole proof we will assume that $p \in(1, \infty)$.

Assume that $L^{\Phi, p}$ satisfies conditions a) and b) but $L^{\Phi, p}$ is not uniformly rotund in every direction. Then (see [16]), there exist $x_{n} \in B\left(L^{\Phi, p}\right), z \in L^{\Phi, p}$ such that $x_{n}+z \in B\left(L^{\Phi, p}\right)$ for any $n \in \mathbb{N},\left\|x_{n}\right\|_{\Phi, p} \rightarrow 1,\left\|x_{n}+z\right\|_{\Phi, p} \rightarrow 1$ and $\left\|2 x_{n}+z\right\|_{\Phi, p} \rightarrow 2$ as $n \rightarrow \infty$ but $z \neq 0$. Since $\Phi$ is a strictly convex $\mathrm{N}$-function, by Corollary $1, \Phi$ is $k_{p}$-unique. Let $K_{p}\left(x_{n}\right)=\left\{k_{n}\right\}$. Without loss of generality we can assume that the sequence $\left(x_{n}\right)_{n=1}^{\infty}$ does not converge to 0 in measure. Indeed, if $x_{n} \stackrel{\mu}{\rightarrow} 0$, then we replace $x_{n}$ and $z$ by $x_{n}^{\prime}=x_{n}+\frac{z}{4}$ and $z^{\prime}=\frac{z}{2}$, respectively. By virtue of Lemma 7 and by the definitions of $x_{n}^{\prime}$ and $z^{\prime}$, we get that $\left\|x_{n}^{\prime}\right\|_{\Phi, p} \rightarrow 1,\left\|x_{n}^{\prime}+z^{\prime}\right\|_{\Phi, p}=\left\|x_{n}+\frac{3}{4} z\right\|_{\Phi, p} \rightarrow 1$ and $\left\|2 x_{n}^{\prime}+z^{\prime}\right\|_{\Phi, p}=\left\|2 x_{n}+z\right\|_{\Phi, p} \rightarrow 2$ as $n \rightarrow \infty$ but $z^{\prime} \neq 0$. Obviously $x_{n}^{\prime} \stackrel{\mu}{\leftrightarrow} 0$. By Lemma 3 , the sequence $\left(k_{n}\right)_{n=1}^{\infty}$ is bounded. Passing to a subsequence, if necessary, we may assume that $k_{n} \rightarrow k(k \geq 1)$ and $1 \leq k_{n} \leq 2 k$ for all $n \in \mathbb{N}$. Moreover, we can assume that $2 k\|z\|_{\Phi, p} \leq 1$ and that $\left(x_{n}+z\right)_{n=1}^{\infty}$ does not converge to zero in measure (otherwise, instead of $z$ we take $\beta z$ for some $\beta>0$ ).

Let $y_{n}=x_{n}+z$ and $K_{p}\left(y_{n}\right)=\left\{h_{n}\right\}$. We can also assume that $h_{n} \rightarrow h$ as $n \rightarrow \infty$ $(h \geq 1)$. Then, by Lemma $6, k_{n} x_{n}-h_{n} y_{n} \rightarrow 0$ in measure and we conclude that $k \neq h$; otherwise we would have that $k_{n} x_{n}-h_{n} y_{n}=k_{n} x_{n}-h_{n} x_{n}-h_{n} z=\left(k_{n}-h_{n}\right) x_{n}-h_{n} z \stackrel{\mu}{\rightarrow} 0$, so if $k=h$, then $z \stackrel{\mu}{\rightarrow} 0$, a contradiction. In what follows, we will consider only the case when $k>h$ (the other case is similar). Without loss of generality, passing to a subsequence if necessary, we may assume that $k_{n}>h_{n}$ for any $n \in \mathbb{N}$ and $k_{n} x_{n}-h_{n} y_{n} \rightarrow 0 \mu$-a.e. on $\Omega$. Set $\lambda_{n}=\frac{h_{n}}{k_{n}+h_{n}}$. Then $\lambda_{n} \rightarrow \frac{h}{k+h}$ as $n \rightarrow \infty$, so $\sigma \leq \lambda_{n} \leq \frac{1}{2}$ for some $\sigma>0$.

Since $z \neq 0$, there is $c>0$ such that $\mu(E)=d>0$, where $E=E_{c}:=\{t \in \Omega:|z(t)|>$ $c$ \}. Let $\varepsilon>0$ be arbitrary. By the assumption b) there exist $A>0$ and $\gamma \in(0,1)$ such that whenever $\tau \geq \varepsilon$ and $p_{+}(\tau) \leq(1+\gamma) p_{+}\left(\frac{1+\sigma \varepsilon}{1+2 \sigma \varepsilon} \tau\right)$, then 


$$
p_{+}\left(\frac{1+\sigma \varepsilon}{1+2 \sigma \varepsilon} \tau\right) \leq A p_{+}\left(\frac{\varepsilon}{2 k} \cdot \frac{1+\sigma \varepsilon}{1+2 \sigma \varepsilon} \tau\right) \leq A p_{+}\left(\frac{\varepsilon}{2 k} \tau\right) .
$$

For such $\sigma, \varepsilon, \gamma$, by Lemma 4, it follows that there exists $\delta>0$ such that for any $u>0$ and $\lambda \in\left[\sigma, \frac{1}{2}\right]$ the inequality

$$
\lambda \Phi((1+\varepsilon) u)+(1-\lambda) \Phi(u) \leq(1+\delta)[\Phi(\lambda(1+\varepsilon) u+(1-\lambda) u)]
$$

implies the existence of $\tau \in[u+\lambda \varepsilon u, u+\varepsilon u]$ satisfying the inequality

$$
p_{+}(\tau) \leq(1+\gamma) p_{+}\left(\frac{1+\sigma \varepsilon}{1+2 \sigma \varepsilon} \tau\right) .
$$

Since $\|2 k z\|_{\Phi, p} \leq 1$, by Lemma 2 , we get that $I_{\Phi}(2 k z) \leq 1$ and $\left\langle|z|, p_{+}(|z|)\right\rangle=I_{\Phi}(z)+$ $I_{\Psi}\left(p_{+}(|z|)\right) \leq 2$. Therefore, by absolute continuity of the integral, there exists $\widetilde{\alpha} \in\left(0, \frac{d}{2}\right)$ such that if $B \subset \Omega$ and $\mu(B) \leq \widetilde{\alpha}$, then

$$
\int_{B}|z(t)| p_{+}(|z(t)|) d t \leq \frac{\varepsilon^{2}}{k A} \text { and } I_{\Phi}\left(2 k z \chi_{B}\right)<\varepsilon .
$$

Since $k_{n} x_{n}-h_{n} y_{n} \rightarrow 0 \mu$-a.e. on $\Omega$, by Theorem 1, passing to subsequence if necessary, we can find $F \in \Sigma$ such that $\mu(\Omega \backslash F)<\widetilde{\alpha}$ and $k_{n} x_{n}-h_{n} y_{n} \rightarrow 0$ uniformly on $F$, and $|z| \leq a_{1}$ on $F$ for some $a_{1}>0$. Notice that $\mu(E \cap F)>\frac{d}{2}$. Indeed,

$$
d=\mu(E)=\mu(E \cap F)+\mu(E \backslash F) \leq \mu(E \cap F)+\mu(\Omega \backslash F)<\mu(E \cap F)+\frac{d}{2} .
$$

Hence $\mu(E \cap F)>\frac{d}{2}$ and we deduce that

$$
I_{\Phi}\left(\frac{h}{k-h} z \chi_{F}\right) \geq I_{\Phi}\left(\frac{h}{k-h} z \chi_{F \cap E}\right) \geq \frac{d}{2} \Phi\left(\frac{h c}{k-h}\right) .
$$

For every $n \in \mathbb{N}$, let us divide $\Omega$ into the following sets:

$$
\begin{aligned}
A_{n}= & \left\{t \in \Omega \backslash F: x_{n}(t) y_{n}(t)<0\right\}, \\
I_{n}= & \left\{t \in \Omega \backslash\left\{F \cup A_{n}\right\}: \max \left\{\left|k_{n} x_{n}(t)\right|,\left|h_{n} y_{n}(t)\right|\right\}<\varepsilon\right\}, \\
J_{n}= & \left\{t \in \Omega \backslash\left\{F \cup A_{n} \cup I_{n}\right\}:\left|k_{n} x_{n}(t)-h_{n} y_{n}(t)\right| \leq \varepsilon \max \left\{\left|k_{n} x_{n}(t)\right|,\left|h_{n} y_{n}(t)\right|\right\}\right\}, \\
H_{n}= & \left\{t \in \Omega \backslash\left\{F \cup A_{n} \cup I_{n} \cup J_{n}\right\}:(1+\delta) \Phi\left(\frac{k_{n} h_{n}}{k_{n}+h_{n}}\left(x_{n}(t)+y_{n}(t)\right)\right)\right. \\
& \left.<\frac{h_{n} \Phi\left(k_{n} x_{n}(t)\right)}{k_{n}+h_{n}}+\frac{k_{n} \Phi\left(h_{n} y_{n}(t)\right)}{k_{n}+h_{n}}\right\}, \\
Q_{n}= & \left\{t \in \Omega \backslash\left\{F \cup A_{n} \cup I_{n} \cup J_{n} \cup H_{n}\right\}:|z(t)|<\varepsilon\left|x_{n}(t)\right| \text { or }\left|x_{n}(t)\right|<\left|y_{n}(t)\right|\right\}, \\
T_{n}= & \Omega \backslash\left\{F \cup A_{n} \cup I_{n} \cup J_{n} \cup H_{n} \cup Q_{n}\right\} .
\end{aligned}
$$

Let us pick $v_{n} \in B\left(L^{\Psi, q}\right)\left(q \in(1, \infty)\right.$ and $\left.\frac{1}{p}+\frac{1}{q}=1\right)$ such that $v_{n}(t)\left[x_{n}(t)+y_{n}(t)\right] \geq 0$ for all $t \in \Omega$ and $\left\langle v_{n}, x_{n}+y_{n}\right\rangle \rightarrow 2$ as $n \rightarrow \infty$. Then, by the linearity of the integral and by the assumptions about $x_{n}$ and $y_{n}=x_{n}+z$, we obtain that $\left\langle v_{n}, x_{n}\right\rangle \rightarrow 1$ and $\left\langle v_{n}, y_{n}\right\rangle \rightarrow 1$ as $n \rightarrow \infty$ and, consequently,

$$
k-h=\lim _{n \rightarrow \infty}\left(k_{n}-h_{n}\right)=\lim _{n \rightarrow \infty} \int_{\Omega} v_{n}(t)\left[k_{n} x_{n}(t)-h_{n} y_{n}(t)\right] d t .
$$

Now, we will find the upper estimates of the integrals $\int\left|\left[k_{n} x_{n}(t)-h_{n} y_{n}(t)\right] v_{n}(t)\right| d t$, where $C$ denotes one of the following sets: $F, A_{n}, I_{n}, J_{n}, \stackrel{C}{H_{n}}, Q_{n}$ and $T_{n}$, respectively. 
Since $k_{n} x_{n}-h_{n} y_{n} \rightarrow 0$ uniformly on $F$, for large $n$, we obtain that

$$
\int_{F}\left|\left[k_{n} x_{n}(t)-h_{n} y_{n}(t)\right] v_{n}(t)\right| d t<\varepsilon .
$$

Notice that if $t \in A_{n}$, then $x_{n}(t) y_{n}(t)<0$, i.e. $x_{n}(t)\left[x_{n}(t)+z(t)\right]<0$, yields that $x_{n}(t) z(t)<0$ and $\left|x_{n}(t)\right|<|z(t)|$. Therefore, for any $A \in \Sigma$ contained in the set $A_{n}$, by (3.8) we obtain that

$$
\begin{aligned}
\int_{A}\left|\Phi\left(k_{n} x_{n}(t)\right)-\Phi\left(h_{n} y_{n}(t)\right)\right| d t & =\int_{A}\left|\Phi\left(k_{n} x_{n}(t)\right)-\Phi\left(h_{n}\left(x_{n}(t)+z(t)\right)\right)\right| d t \\
& \leq \int_{A}\left[\Phi\left(k_{n} z(t)\right)+\Phi\left(h_{n} z(t)\right)\right] d t \\
& \leq 2 \int_{A} \Phi(2 k z(t)) d t<2 \varepsilon .
\end{aligned}
$$

Hence, as well as by virtue of Lemma 5, for $n$ large enough, we get that

$$
\int_{A_{n}}\left|\left[k_{n} x_{n}(t)-h_{n} y_{n}(t)\right] v_{n}(t)\right| d t \leq 2 \varepsilon .
$$

Notice that the Hölder inequality give us that

$$
\begin{aligned}
& \int_{I_{n}}\left|\left[k_{n} x_{n}(t)-h_{n} y_{n}(t)\right] v_{n}(t)\right| d t \leq\left\|\left(k_{n} x_{n}-h_{n} y_{n}\right) \chi_{I_{n}}\right\|_{\Phi, p} \cdot\left\|v_{n}\right\| \Psi, q \\
& \quad \leq 2 \varepsilon\left\|\chi_{\Omega}\right\|_{\Phi, p} \leq \varepsilon 2^{1+\frac{1}{p}}\left\|\chi_{\Omega}\right\|_{\Phi} .
\end{aligned}
$$

By the definition of the set $J_{n}$ and by the conditions that $1 \leftarrow\left\langle v_{n}, x_{n}\right\rangle \leq 1$ and $1 \leftarrow$ $\left\langle v_{n}, y_{n}\right\rangle \leq 1$, we get that

$$
\begin{aligned}
\int_{J_{n}}\left|\left[k_{n} x_{n}(t)-h_{n} y_{n}(t)\right] v_{n}(t)\right| d t & \leq \varepsilon \int_{J_{n}}\left(\left|k_{n} x_{n}(t)\right|+\left|h_{n} y_{n}(t)\right|\right)\left|v_{n}(t)\right| d t \\
& \leq \varepsilon\left(k_{n}+h_{n}\right) .
\end{aligned}
$$

For each set $H \in \Sigma$, being a subset of $H_{n}$, using the method from the proof of Lemma 6 , by continuity of $s_{p}\left(\right.$.) and by the fact that $\frac{k_{n} h_{n}}{k_{n}+h_{n}} \geq \frac{k_{n} h_{n}}{2 k_{n}} \geq \frac{1}{2}$, we conclude that

$$
\begin{aligned}
0 & \leftarrow \frac{h_{n} I_{\Phi}\left(k_{n} x_{n}\right)}{k_{n}+h_{n}}+\frac{k_{n} I_{\Phi}\left(h_{n} y_{n}\right)}{k_{n}+h_{n}}-I_{\Phi}\left(\frac{k_{n} h_{n}}{k_{n}+h_{n}}\left(x_{n}+y_{n}\right)\right) \\
\geq & \frac{h_{n} I_{\Phi}\left(k_{n} x_{n} \chi_{H}\right)}{k_{n}+h_{n}}+\frac{k_{n} I_{\Phi}\left(h_{n} y_{n} \chi_{H}\right)}{k_{n}+h_{n}}-I_{\Phi}\left(\frac{k_{n} h_{n}}{k_{n}+h_{n}}\left(x_{n}+y_{n}\right) \chi_{H}\right) \\
\geq & \frac{1}{2}\left(\frac{I_{\Phi}\left(k_{n} x_{n} \chi_{H}\right)}{k_{n}}+\frac{I_{\Phi}\left(h_{n} y_{n} \chi_{H}\right)}{h_{n}}\right) \\
& -\frac{1}{2}\left(1-\frac{\delta}{1+\delta}\right) \int_{H}\left[\frac{1}{k_{n}} \Phi\left(k_{n} x_{n}(t)\right)+\frac{1}{h_{n}} \Phi\left(h_{n} y_{n}(t)\right)\right] d t \\
= & \frac{\delta}{2(1+\delta)} \int_{H}\left[\frac{1}{k_{n}} \Phi\left(k_{n} x_{n}(t)\right)+\frac{1}{h_{n}} \Phi\left(h_{n} y_{n}(t)\right)\right] d t,
\end{aligned}
$$


whence, for $n$ large enough, we obtain that

$$
\int_{H_{n}}\left|\left(k_{n} x_{n}(t)-h_{n} y_{n}(t)\right) v_{n}(t)\right| d t \leq \varepsilon .
$$

By the facts that $k_{n} x_{n}-h_{n} y_{n} \rightarrow 0$ uniformly on $F$ and $y_{n}=x_{n}+z$, we conclude that $x_{n} \rightarrow \frac{h}{k-h} z$ uniformly on the set $F$. Hence, and by inequality (3.9), we get that

$$
\frac{1}{k_{n}} I_{\Phi}\left(k_{n} x_{n} \chi_{F}\right) \geq I_{\Phi}\left(x_{n} \chi_{F}\right) \rightarrow I_{\Phi}\left(\frac{h}{k-h} z \chi_{F}\right) \geq \frac{d}{2} \Phi\left(\frac{h c}{k-h}\right) .
$$

By the assumption $\left\|x_{n}\right\|_{\Phi, p} \rightarrow 1$ as $n \rightarrow \infty$ and the fact that $k_{n} \in K_{p}\left(x_{n}\right)$, we obtain that $\lim _{n \rightarrow \infty} \frac{1}{k_{n}^{p}}\left(1+I_{\Phi}^{p}\left(k_{n} x_{n}\right)\right)=1$. Consequently, $1+\lim _{n \rightarrow \infty} I_{\Phi}^{p}\left(k_{n} x_{n}\right)=\lim _{n \rightarrow \infty} k_{n}^{p}$, whence

$$
\lim _{n \rightarrow \infty}\left(k_{n}^{p}-I_{\Phi}^{p}\left(k_{n} x_{n}\right)\right)=1 .
$$

But by the superadditivity of the function $g(u)=u^{p}, p>1$, and by (3.15), we get that

$$
\begin{aligned}
\lim _{n \rightarrow \infty} I_{\Phi}^{p}\left(k_{n} x_{n}\right) & =\lim _{n \rightarrow \infty}\left[I_{\Phi}\left(k_{n} x_{n} \chi_{Q_{n}}\right)+I_{\Phi}\left(k_{n} x_{n} \chi_{\Omega \backslash Q_{n}}\right)\right]^{p} \\
& \geq \lim _{n \rightarrow \infty}\left[I_{\Phi}^{p}\left(k_{n} x_{n} \chi_{Q_{n}}\right)+I_{\Phi}^{p}\left(k_{n} x_{n} \chi_{\Omega \backslash Q_{n}}\right)\right] \\
& \geq \lim _{n \rightarrow \infty}\left[I_{\Phi}^{p}\left(k_{n} x_{n} \chi_{Q_{n}}\right)+I_{\Phi}^{p}\left(k_{n} x_{n} \chi_{F}\right)\right] \\
& \geq \lim _{n \rightarrow \infty}\left[I_{\Phi}^{p}\left(k_{n} x_{n} \chi Q_{n}\right)+\left(k_{n} \frac{d}{2} \Phi\left(\frac{h c}{k-h}\right)\right)^{p}\right] .
\end{aligned}
$$

This and (3.16) yield that

$$
\begin{aligned}
1 & =\lim _{n \rightarrow \infty} k_{n}^{p}\left(1-\left(\frac{1}{k_{n}} I_{\Phi}\left(k_{n} x_{n}\right)\right)^{p}\right) \\
& \leq \lim _{n \rightarrow \infty} k_{n}^{p}\left(1-\frac{1}{k_{n}^{p}} I_{\Phi}^{p}\left(k_{n} x_{n} \chi Q_{n}\right)-\left(\frac{d}{2} \Phi\left(\frac{h c}{k-h}\right)\right)^{p}\right) \\
& <\lim _{n \rightarrow \infty} k_{n}^{p}\left(1-\frac{1}{k_{n}^{p}} I_{\Phi}^{p}\left(k_{n} x_{n} \chi Q_{n}\right)-\left(\frac{d}{3} \Phi\left(\frac{h c}{k-h}\right)\right)^{p}\right),
\end{aligned}
$$

whence

$$
\lim _{n \rightarrow \infty} \frac{1}{k_{n}^{p}}\left(1+I_{\Phi}^{p}\left(k_{n} x_{n} \chi Q_{n}\right)\right)<1-\left(\frac{d}{3} \Phi\left(\frac{h c}{k-h}\right)\right)^{p} .
$$

Therefore, for $n$ big enough,

$$
\begin{aligned}
\int_{Q_{n}}\left|x_{n}(t) v_{n}(t)\right| d t & \leq\left\|x_{n} \chi Q_{n}\right\|_{\Phi, p} \leq \frac{1}{k_{n}}\left(1+I_{\Phi}^{p}\left(k_{n} x_{n} \chi_{Q_{n}}\right)\right)^{\frac{1}{p}} \\
& <\left(1-\left(\frac{d}{3} \Phi\left(\frac{h c}{k-h}\right)\right)^{p}\right)^{\frac{1}{p}} .
\end{aligned}
$$

Since $Q_{n} \subset \Omega \backslash\left\{F \cup A_{n}\right\}$, we have that $x_{n}(t) y_{n}(t)=x(t)(x(t)+z(t)) \geq 0$. Since $v_{n}(t)\left[x_{n}(t)+y_{n}(t)\right] \geq 0$, then both $x_{n}(t) v_{n}(t) \geq 0$ and $y_{n}(t) v_{n}(t) \geq 0$ (so $z(t) v_{n}(t) \geq 0$ ). Hence, if $\left|x_{n}(t)\right|<\left|y_{n}(t)\right|$, then $x_{n}(t) z(t)>0$ and, consequently,

$$
\begin{aligned}
v_{n}(t)\left[k_{n} x_{n}(t)-h_{n} y_{n}(t)\right] & =\left(k_{n}-h_{n}\right) x_{n}(t) v_{n}(t)-h_{n} z(t) v_{n}(t) \\
& <\left(k_{n}-h_{n}\right) x_{n}(t) v_{n}(t)
\end{aligned}
$$


and if $|z(t)|<\varepsilon\left|x_{n}(t)\right|$, then

$$
v_{n}(t)\left[k_{n} x_{n}(t)-h_{n} y_{n}(t)\right] \leq\left(k_{n}-h_{n}\right) x_{n}(t) v_{n}(t)+\varepsilon h_{n} x_{n}(t) v_{n}(t) .
$$

Therefore, applying also (3.17) and Lemma 5, for $n$ large enough, we obtain

$$
\begin{aligned}
\int_{Q_{n}}\left(k_{n} x_{n}(t)-h_{n} y_{n}(t)\right) v_{n}(t) d t & \leq\left(k_{n}-h_{n}+\varepsilon h_{n}\right) \int_{Q_{n}} x_{n}(t) v_{n}(t) d t \\
& <\left(k_{n}-h_{n}+\varepsilon h_{n}\right)\left[1-\left(\frac{d}{3} \Phi\left(\frac{h c}{k-h}\right)\right)^{p}\right]^{\frac{1}{p}} .
\end{aligned}
$$

Noticing that $t \notin Q_{n} \cup J_{n} \cup A_{n}$ whenever $t \in T_{n}$, we get that

$$
\varepsilon h_{n}\left|y_{n}(t)\right| \leq \varepsilon k_{n}\left|x_{n}(t)\right| \leq k_{n}\left|x_{n}(t)\right|-h_{n}\left|y_{n}(t)\right|,
$$

i.e. $\frac{k_{n}\left|x_{n}(t)\right|}{h_{n}\left|y_{n}(t)\right|} \geq 1+\varepsilon$. By virtue of Lemma 4 and by the fact that $t \notin H_{n}$, i.e.

$$
\frac{\lambda_{n} \Phi\left(k_{n} x_{n}(t)\right)+\left(1-\lambda_{n}\right) \Phi\left(h_{n} y_{n}(t)\right)}{\Phi\left(\lambda_{n} k_{n} x_{n}(t)+\left(1-\lambda_{n}\right) h_{n} y_{n}(t)\right)} \leq 1+\delta,
$$

there exists

$$
\tau_{n}(t) \in\left[\lambda_{n} k_{n}\left|x_{n}(t)\right|+\left(1-\lambda_{n}\right) h_{n}\left|y_{n}(t)\right|, k_{n}\left|x_{n}(t)\right|\right]
$$

such that

$$
p_{+}\left(\tau_{n}(t)\right) \leq(1+\gamma) p_{+}\left(\frac{1+\sigma \varepsilon}{1+2 \sigma \varepsilon} \tau_{n}(t)\right) .
$$

Noticing that $t \notin I_{n} \cup Q_{n}$ implies $\tau_{n}(t) \geq \lambda_{n} k_{n}\left|x_{n}(t)\right| \geq \sigma \varepsilon$, by (3.20) and (3.7), we have

$$
p_{+}\left(\tau_{n}(t)\right) \leq(1+\gamma) p_{+}\left(\frac{1+\sigma \varepsilon}{1+2 \sigma \varepsilon} \tau_{n}(t)\right) \leq A p_{+}\left(\frac{\varepsilon}{2 k} \tau_{n}(t)\right) .
$$

From $t \notin H_{n} \cup Q_{n}$, by (3.19), (3.21) and the fact that $k_{n} \leq 2 k$ for any $n \in \mathbb{N}$, we conclude that

$$
\begin{aligned}
\lambda_{n} \Phi\left(k_{n} x_{n}(t)\right)+\left(1-\lambda_{n}\right) \Phi\left(h_{n} y_{n}(t)\right) & \leq(1+\delta) \Phi\left(\lambda_{n} k_{n} x_{n}(t)+\left(1-\lambda_{n}\right) h_{n} y_{n}(t)\right) \\
& \leq(1+\delta) \Phi\left(\tau_{n}(t)\right) \leq(1+\delta) \tau_{n}(t) p_{+}\left(\tau_{n}(t)\right) \\
& \leq A(1+\delta) \tau_{n}(t) p_{+}\left(\frac{\varepsilon}{2 k} \tau_{n}(t)\right) \\
& \leq A(1+\delta) k_{n}\left|x_{n}(t)\right| p_{+}\left(\frac{\varepsilon}{2 k} k_{n}\left|x_{n}(t)\right|\right) \\
& \leq A(1+\delta) k_{n}\left|x_{n}(t)\right| p_{+}\left(\varepsilon\left|x_{n}(t)\right|\right) \\
& \leq \frac{1}{\varepsilon} A k(1+\delta)|z(t)| p_{+}(|z(t)|) .
\end{aligned}
$$

This and conditions (3.8) (recall that $\mu(\Omega \backslash F)<\widetilde{\alpha}<\frac{d}{2}$ ) imply

$$
\begin{aligned}
\int_{T_{n}}\left[\lambda_{n} \Phi\left(k_{n} x_{n}(t)\right)+\left(1-\lambda_{n}\right) \Phi\left(h_{n} y_{n}(t)\right)\right] d t & \leq \frac{1}{\varepsilon} A k(1+\delta) \int_{\Omega \backslash F}|z(t)| p_{+}(|z(t)|) d t \\
& \leq(1+\delta) \varepsilon,
\end{aligned}
$$

whence

$$
\begin{aligned}
\frac{1}{k_{n}+h_{n}} \int_{T_{n}}\left[\Phi\left(k_{n} x_{n}(t)\right)+\Phi\left(h_{n} y_{n}(t)\right)\right] d t & \leq \int_{T_{n}}\left[\lambda_{n} \Phi\left(k_{n} x_{n}(t)\right)+\left(1-\lambda_{n}\right) \Phi\left(h_{n} y_{n}(t)\right)\right] d t \\
& \leq(1+\delta) \varepsilon,
\end{aligned}
$$


i.e.

$$
\int_{T_{n}}\left[\Phi\left(k_{n} x_{n}(t)\right)+\Phi\left(h_{n} y_{n}(t)\right)\right] d t \leq\left(k_{n}+h_{n}\right)(1+\delta) \varepsilon .
$$

Hence, by Lemma 5, for all large $n$, we obtain that

$$
\begin{aligned}
\int_{T_{n}}\left|v_{n}(t)\left(k_{n} x_{n}(t)-h_{n} y_{n}(t)\right)\right| d t & \leq \int_{T_{n}}\left[\Phi\left(k_{n} x_{n}(t)\right)+\Phi\left(h_{n} y_{n}(t)\right)\right] d t \\
& \leq\left(k_{n}+h_{n}\right)(1+\delta) \varepsilon .
\end{aligned}
$$

Denoting $v_{n}(t)\left(k_{n} x_{n}(t)-h_{n} y_{n}(t)\right)$ shortly as $w_{n}(t)$, we get that

$$
\begin{aligned}
k-h= & \lim _{n \rightarrow \infty}\left(k_{n}-h_{n}\right)=\lim _{n \rightarrow \infty} \int_{\Omega} v_{n}(t)\left(k_{n} x_{n}(t)-h_{n} y_{n}(t)\right) d t \\
= & \lim _{n \rightarrow \infty}\left(\int_{F} w_{n}(t) d t+\int_{A_{n}} w_{n}(t) d t+\int_{I_{n}} w_{n}(t) d t+\int_{J_{n}} w_{n}(t) d t+\int_{H_{n}} w_{n}(t) d t\right. \\
& \left.+\int_{Q_{n}} w_{n}(t) d t+\int_{T_{n}} w_{n}(t) d t\right)
\end{aligned}
$$

whence, as well as by inequalities (3.10), (3.11), (3.12), (3.13), (3.14), (3.18) and (3.22) and by the arbitrariness of $\varepsilon>0$, we conclude that

$$
k-h \leq(k-h)\left[1-\left(\frac{d}{3} \Phi\left(\frac{h c}{k-h}\right)\right)^{p}\right]^{\frac{1}{p}},
$$

a contradiction, which shows that $z=0$ a.e. in $\Omega$.

Corollary 2 If $\Phi$ is a strictly convex $N$-function satisfying the $\Delta_{2}(\infty)$-condition, then the Orlicz space $\left(L^{\Phi},\|\cdot\|_{\Phi, p}\right), p \in[1, \infty)$ equipped with the $p$-Amemiya norm and built over a non-atomic finite measure space is uniformly rotund in every direction.

Proof Assume that $\Phi \in \Delta_{2}(\infty)$. Then for any $a>1$ we can find $K>a$ such that $\Phi(2 a u) \leq K \Phi(u)$ for all $u$ large enough, whence

$$
a p_{+}(a u) \leq \frac{\Phi(2 a u)}{u} \leq K \frac{\Phi(u)}{u} \leq K p_{+}(u)
$$

for all $u$ large enough, so by strict convexity of $\mathrm{N}$-function $\Phi$ and by Theorem 4 , we conclude that Orlicz space with the p-Amemiya norm is uniformly rotund in every direction.

At the end, let us recall that the uniform rotundity in every direction plays an important role in the fixed point theory. Namely, every uniformly rotund in every direction Banach space $(X,\|\|$.$) has normal structure (see for example [8]). Consequently, it has the weak$ fixed point property (we refer, for instance, to [8], [21] or [31] for the suitable definitions and results). By this, and by Theorem 4, we get the following 
Corollary 3 Let $(\Omega, \Sigma, \mu)$ be a non-atomic finite and complete measure space and let $p \in[1, \infty)$. Then the Orlicz space $\left(L^{\Phi},\|\cdot\|_{\Phi, p}\right)$ equipped with the $p$-Amemiya norm and generated by a strictly convex $N$-function $\Phi$ satisfying the $\Delta_{2}(\infty)$-condition has the weak fixed point property.

Open Access This article is distributed under the terms of the Creative Commons Attribution 4.0 International License (http://creativecommons.org/licenses/by/4.0/), which permits unrestricted use, distribution, and reproduction in any medium, provided you give appropriate credit to the original author(s) and the source, provide a link to the Creative Commons license, and indicate if changes were made.

\section{References}

1. Akimovič, V.A.: The uniform convexity and uniform smoothness of Orlicz spaces. Teor. Funkciŭ Funkcional. Anal. i Priložen 15, 114-121 (1972)

2. Cao, F., Mao, R., Wang, B.: Uniform rotundity in every direction of Orlicz-Sobolev spaces. J. Inequal. Appl. 2016, 277 (2016)

3. Chen, S.: Geometry of Orlicz spaces. Diss. Math. 356, 1-204 (1996)

4. Chen, S.T.: On the properties of locally K-uniform rotundity and uniform rotundity in every direction for Banach spaces, (Chinese). Nat. Sci. J. Harbin Normal Univ. 3(3), 1-7 (1987)

5. Chen, S.T.: Some rotundities of Orlicz spaces with Orlicz norm. Bull. Pol. Acad. Sci. Math. 34(9-10), 585-596 (1986)

6. Chen, L., Cui, Y.: Complex extreme points and complex rotundity in Orlicz function spaces equipped with the p-Amemiya norm. Nonlinear Anal. 73(5), 1389-1393 (2010)

7. Chen, L., Cui, Y.: Complex rotundity of Orlicz sequence spaces equipped with the p-Amemiya norm. J. Math. Anal. Appl. 378(1), 151-158 (2011)

8. Chen, S., Cui, Y., Hudzik, H., Sims, H.: Geometric properties related to fixed point theory in some Banach function lattices. In: Kirk, W.A., Sims, B. (eds.) Handbook of Metric Fixed Point Theory, pp. 339-389. Kluwer Academic Publishers, Dordrecht (2001)

9. Chen, L., Cui, Y., Zhao, Y.: Complex convexity of Musielak-Orlicz function spaces equipped with the p-Amemiya norm. Abstr. Appl. Anal. (2014). Art. ID 190203

10. Chen, L.L., Cui, Y.A., Zhao, Y.F., Niu, J.L.: Complex convexity of Musielak-Orlicz sequence spaces equipped with the p-Amemiya norm, (Chinese). Acta Math. Sin. (Chin. Ser.) 58(1), 169-176 (2015)

11. Cui, Y., Duan, L., Hudzik, H., Wisła, M.: Basic theory of $p$-Amemiya norm in Orlicz spaces $(1 \leq p \leq \infty)$ : extreme points and rotundity in Orlicz spaces endowed with these norms. Nonlinear Anal. 69(5-6), 1796$816(2008)$

12. Cui, Y., Hudzik, H., Wisła, M.: M-Constants, Dominguez-Benavides coefficient, and weak fixed point property in Orlicz sequence spaces equipped with the p-Amemiya norm. Fixed Point Theory Appl. 2016, 89 (2016)

13. Cui, Y., Hudzik, H., Wisła, M.: Monotonicity properties and dominated best approximation problems in Orlicz spaces equipped with the p-Amemiya norm. J. Math. Anal. Appl. 432(2), 1095-1105 (2015)

14. Cui, Y., Hudzik, H., Li, J., Wisła, M.: Strongly extreme points in Orlicz spaces equipped with the pAmemiya norm. Nonlinear Anal. 71, 6343-6364 (2009)

15. Cui, Y., Hudzik, H., Wisła, M., Wlaźlak, K.: Non-squareness properties of Orlicz spaces equipped with the p-Amemiya norm. Nonlinear Anal. 75(10), 3973-3993 (2012)

16. Day, M.M., James, R.C., Swaminathan, S.: Normed linear spaces that are uniform convexity in every direction. Can. J. Math. 23, 1051-1059 (1971)

17. Duan, L.F., Xu, J., Cui, Y.: Extreme points and rotundity in Orlicz sequence spaces equipped with pAmemiya $(1 \leq p \leq \infty)$ norm, (in Chinese). J. Jilin Univ. Sci. 50(5), 902-906 (2012)

18. Garkavi, A.L.: On the Čebyšev center of a set in a normed space (Russian). In: Studies of Modern Problems of Constructive Theory of Functions, pp. 328-331. Fizmatgiz, Moscow (1961)

19. Garkavi, A.L.: The best possible net and the best possible cross-section of a set in a normed space (Russian). Izv. Akad. Nauk SSSR Ser. Mat. 26, 87-106 (1962)

20. Garkavi, A.L.: The best possible net and the best possible cross-section of a set in a normed space (Russian). Am. Math. Soc. Transl. II. Ser. 39, 111-132 (1964)

21. Goebel, K., Kirk, W.: Topics in Metric Fixed Point Theory. Cambridge University Press, Cambridge (1991) 
22. Halmos, P.R.: Measure Theory. D. Van Nostrand Company Inc, New York (1950)

23. He, X., Cui, Y., Hudzik, H.: The fixed point property of Orlicz sequence spaces equipped with the pAmemiya norm. Fixed Point Theory Appl. 2013, 340 (2013)

24. He, X., Yu, J., Cui, Y., Huo, X.: Packing constant in Orlicz sequence spaces equipped with the p-Amemiya norm. Abstr. Appl. Anal. (2014). Art. ID 626491

25. Hudzik, H., Maligranda, L.: Amemiya norm equals Orlicz norm in general. Indag. Math. 11(4), 573-585 (2000)

26. Kaczmarek R.: Uniform rotundity of Orlicz function spaces equipped with the $p$-Amemiya norm. Mathematische Nachrichten. 1-19 (2018). https://doi.org/10.1002/mana.201700025

27. Kamińska, A.: On some convexity properties of Musielak-Orlicz spaces. In: Proceedings of the 12th Winter School on Abstract Analysis (Srní, 1984). Rend. Circ. Mat. Palermo (2) 1984, Suppl. No. 5, pp. 63-72 (1984)

28. Kamińska, A.: Some convexity properties of Musielak-Orlicz spaces of Bochner type. In: Proceedings of the 13th Winter School on Abstract Analysis (Srní, 1985). Rend. Circ. Mat. Palermo (2) Suppl. No. 10 (1985), pp. 63-73 (1986)

29. Kamińska, A.: Uniform rotundity in every direction of sequence Orlicz spaces. Bull. Pol. Acad. Sci. Math. 32(9-10), 589-594 (1984)

30. Krasnoselskiı̌, M.A., Ya, B.: Rutickiı̌, Convex Functions and Orlicz Spaces, Groningen, Nordhoff, 1961 (English translation); Original Russian edition: Gos. Izd. Fiz. Mat. Lit., Moskva (1958)

31. Kirk, W.A., Sims, B. (eds.): Handbook of Metric Fixed Point Theory. Kluwer Academic, Dordrecht (2001)

32. Kolwicz, P., Płuciennik, R.: On uniform rotundity in every direction in Calderón-Lozanovskiu sequence spaces. J. Convex Anal. 14(3), 621-645 (2007)

33. Li, J., Cui, Y., Hudzik, H., Wisła, M.: Strongly extreme points in Orlicz spaces equipped with the $p$ Amemiya norm. Nonlinear Anal. 71(12), 6343-6364 (2009)

34. Luxemburg, W.A.J.: Banach Function Spaces. Thesis Delft (1955)

35. Maligranda, L.: Orlicz Spaces and Interpolation, (Seminárias de Mathemática: vol. 5), Campinas (Brazil) Univ. Estadual de Campinas (1989)

36. Musielak, J.: Orlicz spaces and modular spaces. In: Lecture Notes Math, vol. 1034. Springer, Berlin (1983)

37. Orlicz, W.: A note on modular spaces. Bull. Acad. Polon. Sci. Math. 9, 157-162 (1961)

38. Orlicz, W.: Über eine gewisse Klasse von Räumen vom Typus B. Bull. Int. Pol. Ser. A 8(9), 207-220 (1932)

39. Rao, M.M., Ren, Z.D.: Theory of Orlicz Spaces. Marcel Dekker Inc., New York (1991)

40. Reisner, S.: On two theorems of Lozanovskir concerning intermediate Banach lattices, in: geometric aspects of functional analysis (Israel GAFA Seminar, 1986/87). In: Lecture Notes in Mathematics, vol. 1317, pp. 67-83. Springer, Berlin (1988)

41. Shi, S.J., Chen, S.T.: Uniform rotundity in every direction of Musielak-Orlicz spaces $L_{p}(x)(\Omega)$ (Chinese). Nat. Sci. J. Harbin Normal Univ. 22(5), 1-3 (2006)

42. Smith, M.A., Turett, B.: Rotundity in Lebesgue-Bochner function spaces. Trans. Am. Math. Soc. 251(1), $105-118(1980)$

43. Wang, T.F., Shi, Z.R., Chen, G.H.: Orlicz sequence spaces endowed with Orlicz norm that are uniformly rotund in every direction. Acta Sci. Math. (Szeged) 59(1-2), 195-208 (1994)

44. Wang, T.F., Shi, Z.R., Cui, Y.: Orlicz spaces that are uniformly rotund in every direction. Comment. Math. (Prace Mat.) 35, 245-262 (1995)

45. Wisła, M.: Geometric properties of Orlicz spaces equipped with p-Amemiya norms-results and open questions. Comment. Math. 55(2), 183-209 (2015)

46. Zizler, V.: On some rotundity and smoothness properties of Banach spaces. Dissertationes Math. Rozprawy Mat., vol. 87 (1971). (errata insert) 